\title{
FLOW CONTROL WITH AEROSPIKE BEHIND BLUFF BODY
}

\author{
SHER AFGHAN KHAN ${ }^{1}$, MOHAMMAD ASADULLAH ${ }^{2}$, \\ FHARUKH AHMED G $\mathbf{M}^{3}$,AHMED JALALUDDEEN ${ }^{4}$ \\ \& MAUGHAL AHMED ALI BAIG 5 \\ ${ }^{1,2}$ Department of Mechanical Engineering, Faculty of Engineering, International \\ Islamic University Malaysia, Kuala Lumpur, Malaysia \\ ${ }^{3}$ Department of Mechanical Engineering, Bearys Institute of Technology, Mangalore, Karnataka, India \\ ${ }^{3}$ Department of Mechanical Engineering, Government Engineering College, Karnataka, India \\ ${ }^{4}$ Department of Mechanical Engineering, Bearys Institute of Technology, Mangaluru, Karnataka, India \\ ${ }^{5}$ Department of Mechanical Engineering, CMR Technical Campus, Hyderabad, Telangana, India
}

Flow control in high-speed is challenging due to the high-pressure shock and low-pressure recirculation bubble attached around the vehicle. Wave drag, and Base drag are important accouterments of flow around high-speed objects. This paper deals with base drag, only and presents an experimental study of aerospikes behind the base of bluff bodies to control this drag. A plate of $1 \mathrm{~mm}$ thickness with two spikes at $11.5 \mathrm{~mm}$ is placed between the nozzle and duct as a passive controller. The Mach numbers deployed for the subsonic regime were 0.6, 0.7 and for transonic regime were $0.8,0.9$ for area ratio 6.25. The $L / W$ ratio taken were $4 \mathrm{~W}, 6 \mathrm{~W}, 8 \mathrm{~W}$ and $10 \mathrm{~W}$. Aerospikes were found to be very effective in controlling base pressure in the transonic regime without influencing the main flow field.

KEYWORDS: Base Pressure, Wall Pressure \& Mach Number
\end{abstract}

Received: May 04, 2018; Accepted: May 25, 2018; Published: Jun 14, 2018; Paper Id: IJMPERDJUN2018106

\section{INTRODUCTION}

Creatures of nature, after years of evolution, reduce drag by biological structural adaptive control [1]. Drag reduction techniques mimicked by us in today's world is a primary engineering goal [2]. The high Mach number vehicles are designed to have bluff-nose to reduce aerodynamic heating but leads to strong bow shock at nose which further lead to high pressure drag. This high-pressure drag is known as wave drag. Another type of pressure drag is base drag i.e. partial vacuum behind the base. The vacuum acting behind the high-speed vehicle can result in $50 \%$ of the total drag of a missile in no-jet condition [3]. This encourages researchers in aerodynamics to explore new techniques to reduce base drag. We, in this paper, have focused on base drag reduction. Device to reduce base drag of wings using blunt base is one of the applications [4]. Another application is launch vehicles [5]. Computational analysis of the effect of geometric and flow parameters on velocity was studied by[6] thus giving future trend for a shift from expensive experimentation to computation. Turbulent flow study after the sudden change in cross-section in subsonic regime is another research area [7]. Thus, we can see the application in different fields and would like a robust control to adapt to the need. 


\section{LITERATURE REVIEW}

\section{Previous Study About Aerospike}

Wave drag reduction using aerospikes at high-speed flows were investigated a long time back [8]. [9] studies force and moments over the blunt-nosed body over the aerospike at Mach number of 6. Laser pulse in the form of acting spike was used to control wave drag [10]. Thus wave losses across a high-pressure along a single normal shock is replaced by pressure distribution along oblique and normal shock [11]. Research shows different types of spikes such as hemisphere spike, conical spike, flat spike, and square spike used to control wave drag and the wake-region behind the fore body is directly influenced by the pressure on its nose and the deflection of the flow [12]. However, all the previous studies focused on controlling high pressure drag using aerospace but no literature to the author's knowledge about base drag reduction with spikes was available. Therefore, in the present work, an attempt is made in controlling depression behind the vehicle using aerospikes. Various methods to reduce drag have been used both to the authors knowledge aerospikes to control base pressure for reducing base drag has been used for the first time.

\section{Previous Study about Base Drag Behind Bluff Bodies}

Manipulation of base pressure can be done by either passive or active control to reduce base drag. Some of the common passive methods are splitter plate, splitter wedges, boat tailing and serrated trailing edge [13] .While active methods are injection [14], base-bleed [15], periodic suction and injection [16], counter flow blowing [17], microjets [18], plasma actuator [19] are being studied by few researchers. [20] investigated different passive and active methods for drag reduction behind bluff bodies but the study was limited to incompressible subsonic flow. [21] used active control of blowing to reduce base drag in axisymmetric bluff bodies. Taking Ahmed body as bluff body, the dragged was reduced with flaps on Ahmed body [22]. Thus, enormous literature can be found regarding base drag reduction behind bluff bodies and aerospikes as a controller in high-pressure zone but nowhere we can find the use of spikes in low-pressure zone. We propose to use aerospikes behind the bluff bodies as shown in the Figure 1.

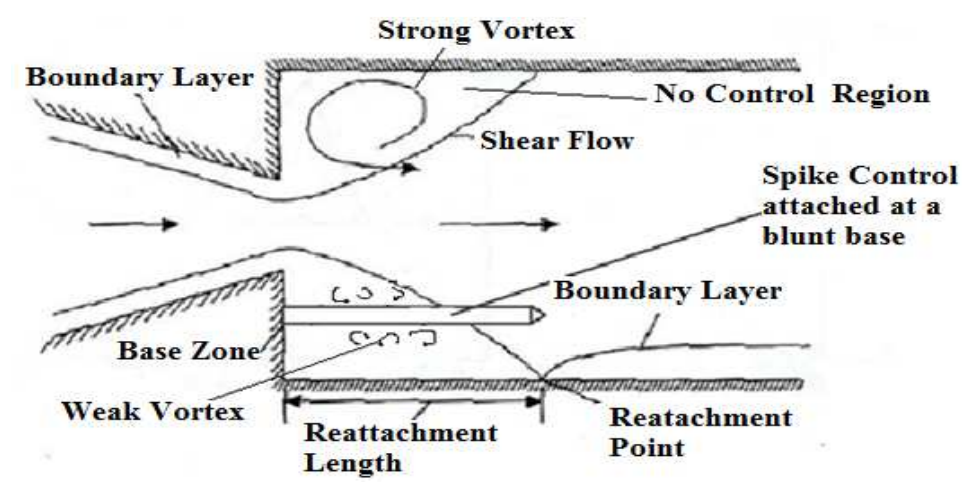

Figure 1: Spike Control Attached Behind the Bluff Body

\section{EXPERIMENTAL SETUP}

\section{Internal Flow Apparatus}

Storage tank with compressed dry air is tunneled to the settling chamber through a control valve. In the settling chamber the flow attainment equilibrium and is further expanded through the nozzles of exit area $100 \mathrm{~mm}^{2}$ to the abrupt duct of cross-section $625 \mathrm{~mm}^{2}$. The desired stagnation pressure in the settling chamber is achieved by regulating the 
control valve. The L/W ratios varied are 4, 6, 8 and 10 for fixed Mach number as 0.6, 0.7, 0.8 and 0.9.

Aerospike control was tested for all ducts from $4 \mathrm{~W}$ to $10 \mathrm{~W}$ and for all Mach numbers as stated above. Pressure transducer PSI model 9205 is used for measuring base, wall and stagnation pressure in the settling chamber. It has 16 channels and pressure range is approximately 0-150 psi. It averages 250 samples per seconds and displays pressure readings from all the channels simultaneously in a window type display on the computer screen. LabVIEW software is used as an interface between DAQ, Sensors and computer. Our focus is mainly on how the geometric parameter L/W ratio influences the base pressure.

\section{Fabricated Models}

The models of the present study consist of area ratio of square duct of a larger cross-sectional area of the square nozzle equal to 6.25.. The convergent nozzle is fabricated out of brass and ducts from a low-grade steel pipe. Two pressure sensing points of $1 \mathrm{~mm}$ diameter at the opposite ends as shown in figure 3 for nozzle and figure 5 for control plate of $1 \mathrm{~mm}$ thickness need to be aligned accurately before starting the experiment to get an accurate base pressure measurement. Also, two aerospikes of the same length to control two separate bubbles have been shown. The diameter of the spike is $2 \mathrm{~mm}$ and length is $8 \mathrm{D}$. In the center of control plate there is a slot of cross-section equal to nozzle exit. Figure 4 displays the four ducts of $4 \mathrm{~W}, 6 \mathrm{~W}, 8 \mathrm{~W}$ and $10 \mathrm{~W}$ respectively, along with pressure tabs for measuring wall pressure. Pressures are measured in various $\mathrm{L} / \mathrm{W}$ ratios at different tab positions on the duct.

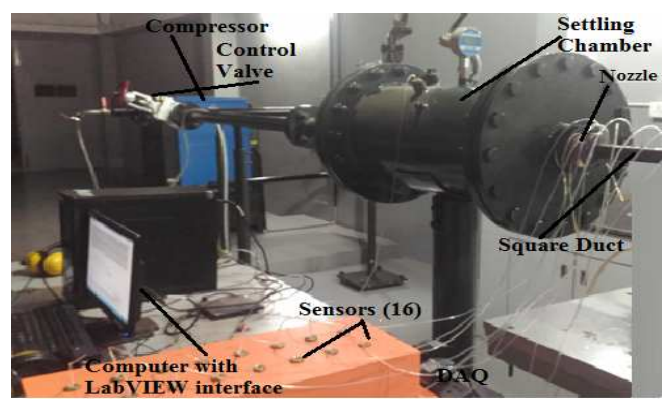

Figure 2: Experimental Setup

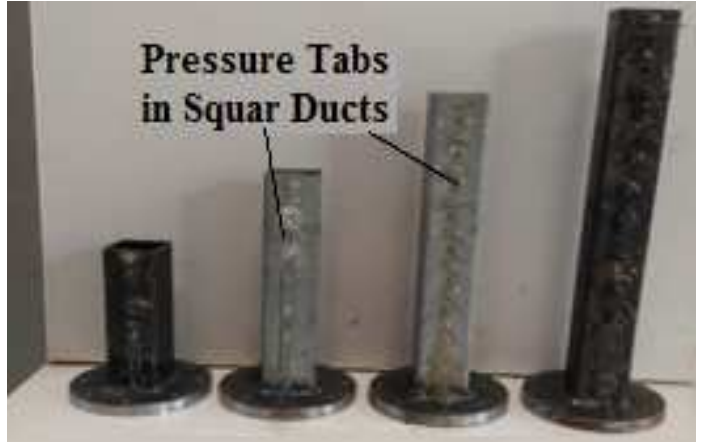

Figure 4: Different L/W Ratio Duct

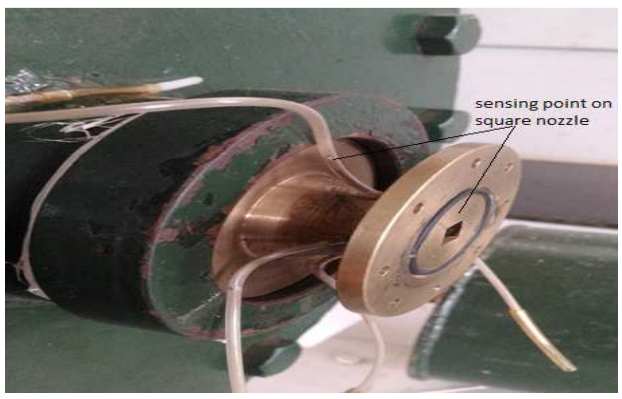

Figure 3: Square Nozzle Attached to Settling Chamber

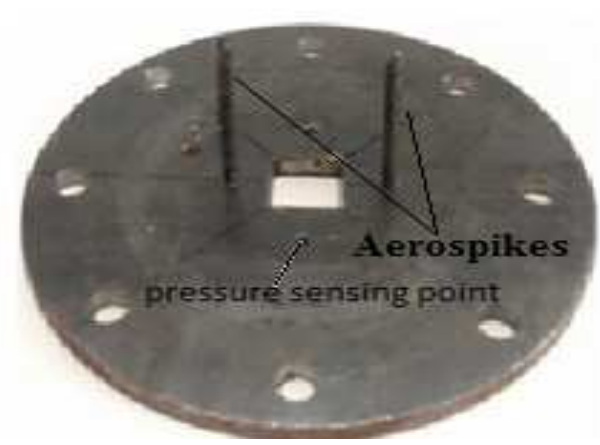

Figure 5: Control Plate with Aerospikes

\section{RESULTS AND DISCUSSIONS}

\section{Base Pressure Analysis}

The focus of this study is to check the effect of aerospikes in controlling the base pressure to reduce base drag for different length of the ducts. From the plots, base pressure clearly shows its dependency on geometric parameter such as 
$\mathrm{L} / \mathrm{W}$ ratio at fixed Mach number and area ratio. Also, the aerospike effect on manipulating base pressure to reduce base drag was quite remarkable for all $\mathrm{L} / \mathrm{W}$ ratios at the constant Mach number and area ratio. The effectiveness may be explained, since control might seem to break large,strong vortex into small, weak vortices as illustrated in figure 1. Thus, decreasing the suction effect and reducing the overall size of recirculation zone. Figure 6-17 shows an increasing trend of base pressure with axial length of the duct without spike control until $\mathrm{L} / \mathrm{W}=6$ and then a decrease until $\mathrm{L} / \mathrm{W}=8$ and then again goes on increasing. For low $\mathrm{L} / \mathrm{D}$ ratio equal to 4 , the control is not affected. This may be due to the small size of duct open to atmosphere, but a reversal takes place and base pressure gradually increases and the control is very positive with increasing L/W ratio for fixed Mach no. at constant area ratio.

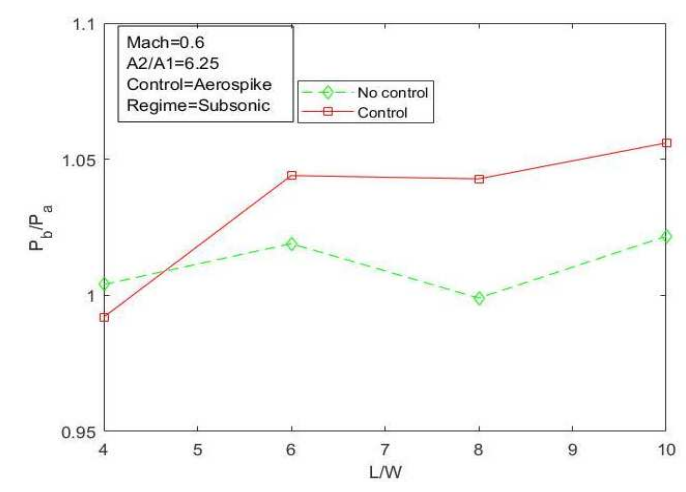

Figure 6: Base Pressure Variation with $\mathrm{L} / \mathrm{W}$ Ratio at $\mathrm{M}=\mathbf{0 . 6}$

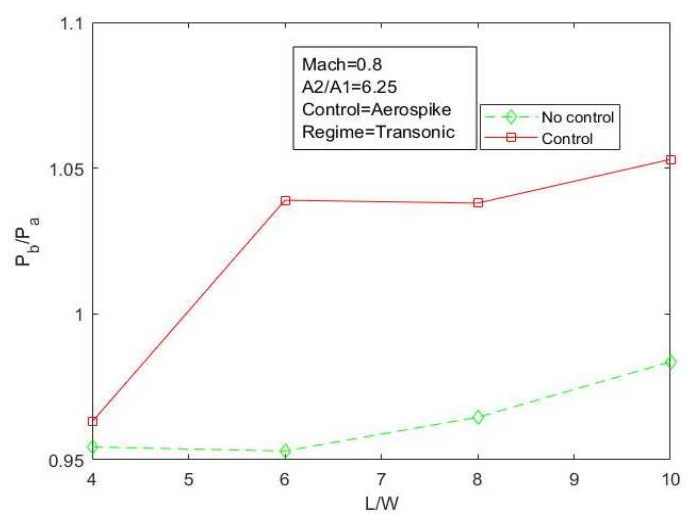

Figure 8: Base Pressure Variation with $L / W$ Ratio at $M=0.8$

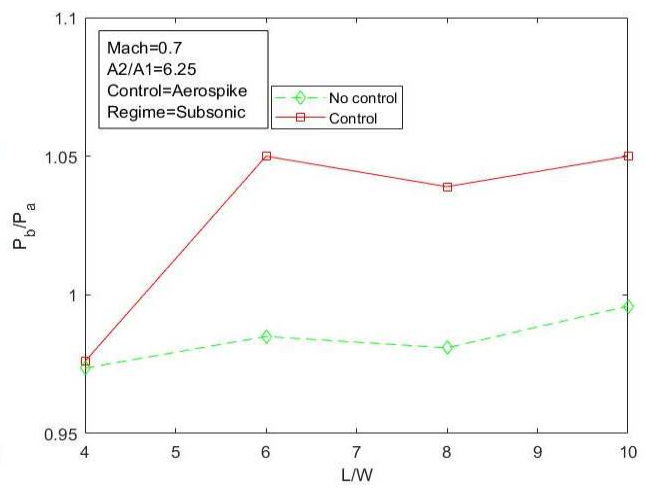

Figure 7: Base Pressure Variation with $\mathrm{L} / \mathrm{W}$ Ratio at $\mathrm{M}=\mathbf{0 . 7}$

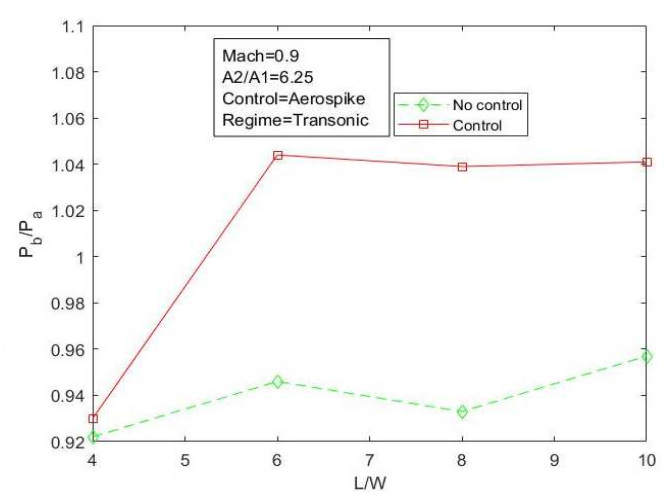

Figure 9: Base Pressure Variation with $L / W$ Ratio at $M=0.9$

\section{Wall Pressure Analysis}
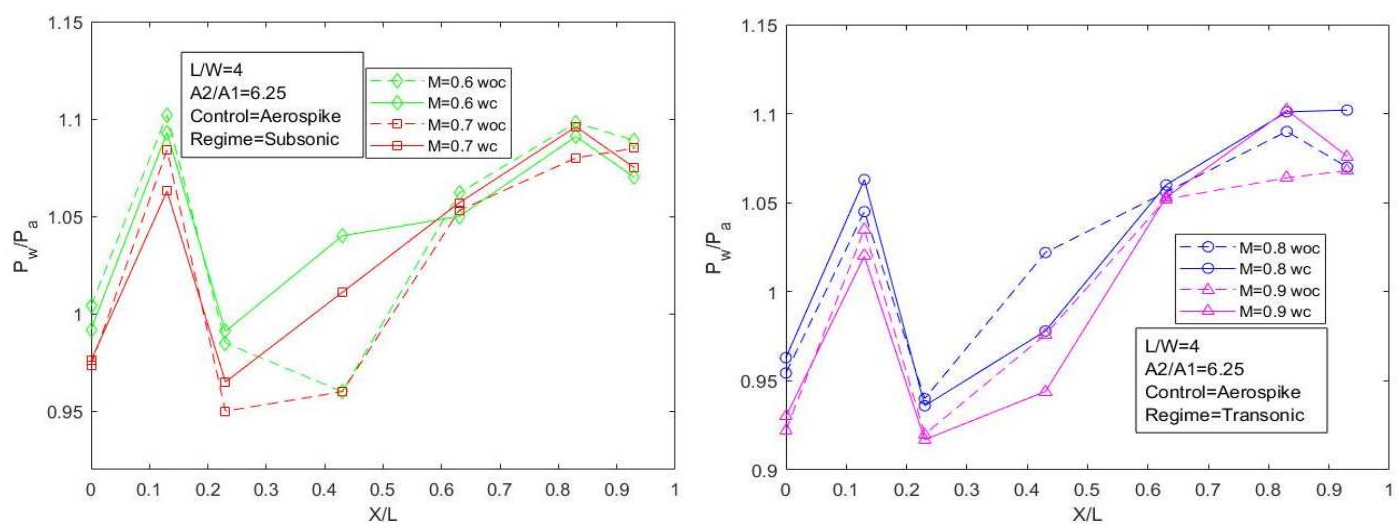
Figure 10: Wall Pressure Variation with $X / L$ at $L / W=4$

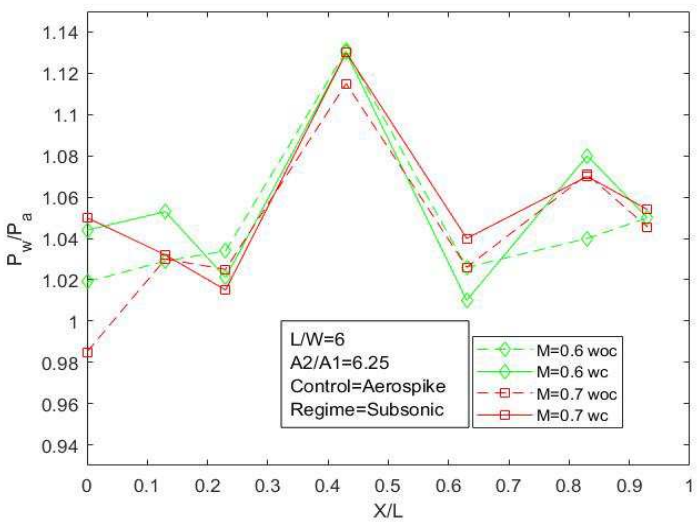

Figure 12: Wall Pressure Variation with $X / L$ at $L / W=6$

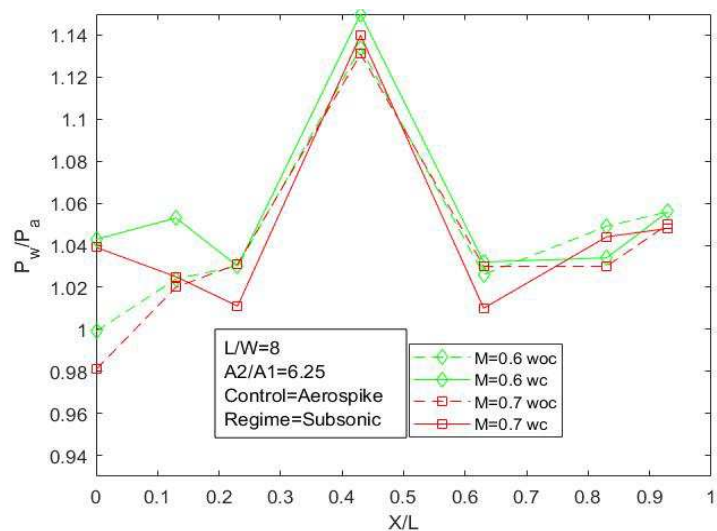

Figure 14: Wall Pressure Variation with $\mathrm{X} / \mathrm{L}$ at $\mathrm{L} / \mathrm{W}=8$

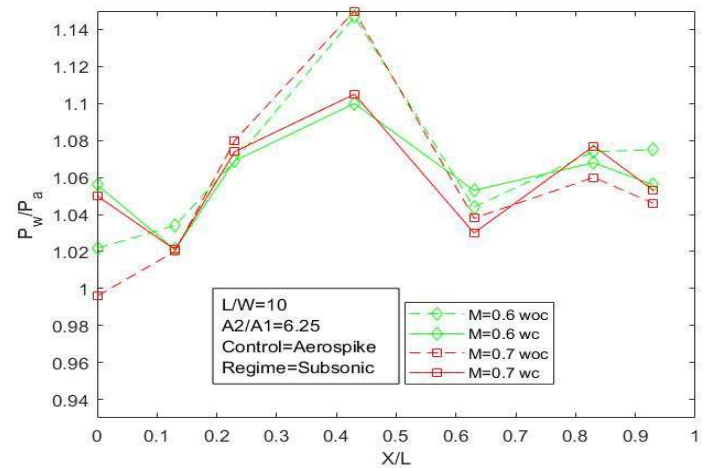

Figure 16: Wall pressure variation with $\mathrm{X} / \mathrm{L}$ at $\mathrm{L} / \mathrm{W}=10$
Figure 11: Wall Pressure Variation with $X / L$ at $L / W=4$

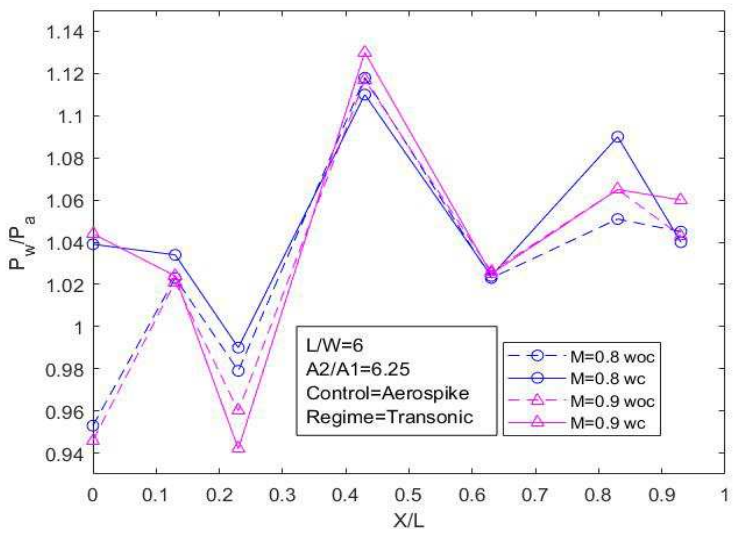

Figure 13: Wall Pressure Variation with $X / L$ at $L / W=6$

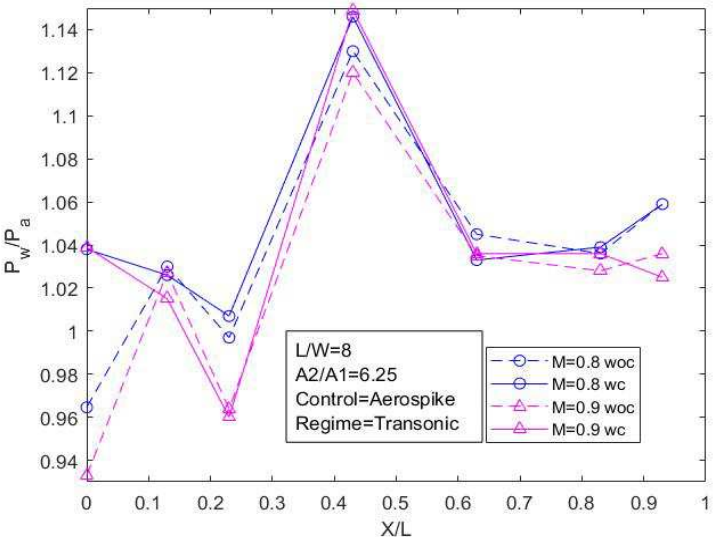

Figure 15: Wall Pressure Variation with $\mathrm{X} / \mathrm{L}$ at $\mathrm{L} / \mathrm{W}=8$

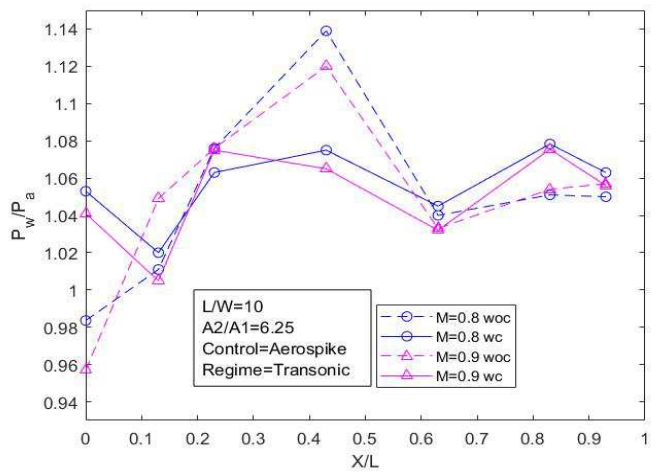

Figure 17: Wall pressure variation with $\mathrm{X} / \mathrm{L}$ at $\mathrm{L} / \mathrm{W}=\mathbf{1 0}$

\section{CONCLUSIONS}

We conclude for a wide range of L/D ratio for fixed Mach numbers and area ratio the following,

- The base pressure is strongly influenced by geometric parameters such as a L/D ratio for a given Mach number for compressible subsonic flow 
- From experimental results, the aerospikes are very effective in controlling base pressure for all L/W ratios. As the length of the duct increases gradually, the control is getting very effective.

- $\quad$ The optimum length where control was most effective seems to be $\mathrm{L} / \mathrm{W}=6$.

- $\quad$ For wall pressure distribution does not adversely affect the flow field for with and without control cases.

- Further research can be carried out by testing aerospikes for supersonic regimes.

\section{ACKNOWLEDGMENT}

The authors acknowledge and thank Bearys Institute of Technology, Mangalore, India for utilizing their research facility in supersonic aerodynamics laboratory.

\section{REFERENCES}

1. G. Yunqing, L. Tao, M. Jiegang, S. Zhengzan, and Z. Peijian, "Analysis of Drag Reduction Methods and Mechanisms of Turbulent," Applied bionics and biomechanics, vol. 2017, 2017.

2. M. Gad-el-Hak, "Flow Control: Passive, Active, and Reactive Flow Management. 2000," ed: Cambridge University Press.

3. P. Viswanath, "Flow management techniques for base and afterbody drag reduction," Progress in Aerospace Sciences, vol. 32, pp. 79-129, 1996.

4. M. Tanner, "A method for reducing the base drag of wings with blunt trailing edge," The Aeronautical Quarterly, vol. 23, pp. 15-23, 1972.

5. J. W. Naughton, "Reduction of Base Drag on Launch Vehicles," 2002.

6. K. A. Pathan, S. Khan, and P. Dabeer, "CFD analysis of effect of Mach number, area ratio and nozzle pressure ratio on velocity for suddenly expanded flows," in Convergence in Technology (I2CT), 2017 2nd International Conference for, 2017, pp. 1104-1110.

7. S. Kangovi and R. Page, "The Turbulent flow through a sudden enlargement at subsonic speeds," in 6th Australasian Hydraulics and Fluid Mechanics Conference. Adelaide, 1977, pp. 213-216.

8. Z. Eghlima and K. Mansour, "Drag reduction for the combination of spike and counterflow jet on blunt body at high Mach number flow," Acta Astronautica, vol. 133, pp. 103-110, 2017.

9. R. Kalimuthu, R. C. Mehta, and E. Rathakrishnan, "Drag reduction for spike attached to blunt-nosed body at Mach 6," Journal of Spacecraft and Rockets, vol. 47, pp. 219-222, 2010.

10. J.-H. Kim, A. Matsuda, T. Sakai, and A. Sasoh, "Wave drag reduction with acting spike induced by laser-pulse energy depositions," AIAA journal, vol. 49, pp. 2076-2078, 2011.

11. M. Rein, H. Rosemann, and E. Schülein, "Wave drag reduction by means of aerospikes on transonic wings," in Shock Waves, ed: Springer, 2009, pp. 1309-1313.

12. S. Khurana, K. Suzuki, and E. Rathakrishnan, "Flow field behavior with Reynolds number variance around a spiked body," Modern Physics Letters B, vol. 30, p. 1650362, 2016.

13. M. Tanner, "Reduction of base drag," Progress in Aerospace Sciences, vol. 16, pp. 369-384, 1975.

14. M. Badrinarayanan, "An experimental investigation of base flows at supersonic speeds," The Aeronautical Journal, vol. 65, pp. 475-482, 1961. 
15. T. Mathur and J. C. Dutton, "Base-bleed experiments with a cylindrical afterbody in supersonic flow," Journal of Spacecraft and Rockets, vol. 33, pp. 30-37, 1996.

16. D. Williams and C. Amato, "Unsteady pulsing of cylinder wakes," in Frontiers in experimental fluid mechanics, ed: Springer, 1989, pp. 337-364.

17. E. Josyula, M. Pinney, and W. B. Blake, "Applications of a counterflow drag reduction technique in high-speed systems," Journal of Spacecraft and Rockets, vol. 39, pp. 605-614, 2002.

18. S. A. Khan and E. Rathakrishnan, "Control of suddenly expanded flows with micro-jets," International journal of Turbo and Jet engines, vol. 20, pp. 63-82, 2003.

19. Adegbola, A. A and Salau, T. A. O (2013). " Characterization of Fluid Flow Using Discrete Vortex Modelling". 2(4). pp 99-108

20. M. Kühn, M. Kühn-Kauffeldt, J. Schein, and A. Belinger, "Plasma actuators for active flow control based on a glow discharge," in Journal of Physics: Conference Series, 2017, p. 012007.

21. G. Buresti, G. V. Iungo, and G. Lombardi, "Methods for the drag reduction of bluff bodies and their application to heavy roadvehicles," 1st Interim Report Contract between CRF and DIA, DDIA, vol. 6, p. 2007, 2007.

22. A. Shimizu, "Drag and wake modification of axisymmetric bluff bodies using coanda blowing," J Jpn Hydraulics Pneumatics Soc, vol. 14, p. 488, 1983.

23. J. Tian, Y. Zhang, H. Zhu, and H. Xiao, "Aerodynamic drag reduction and flow control of Ahmed body with flaps," Advances in Mechanical Engineering, vol. 9, p. 1687814017711390, 2017. 
